\title{
SINDROME DE GARCIN CAUSADA POR ANEURISMA GIGANTE DA ARTÉRIA CARÓTIDA INTERNA
}

\author{
José Pindaro P. Plese * \\ Milberto ScafF ** \\ Walter Carlos Pereira** \\ JosÉ ZACLIS ***
}

A maioria dos aneurismas intracranianos se manifesta por hemorragia subaracnóidea. A esta se somam, com relativa freqüência, sinais de compressão de nervos cranianos, mormente do motor ocular comum. Excepcionalmente, contudo, o aneurisma pode atingir grande volume sem provocar quadro hemorrágico, determinando, porém, compressão de várias estruturas intracranianas. Comporta-se, nestes casos, como processo tumoral, cuja sintomatologia depende de sua topografia e dimensões $1,2,7,15,16,17,18,22,24,25,33$.

A designação aneurisma gigante é empregada quando seu maior eixo ultrapassa $2,5 \mathrm{~cm}^{2}, 5,19,20,24,26,28,29$.

A síndrome de Garcin, como manifestação de aneurisma gigante, não foi ainda relatada na literatura, o que justifica o registro do presente caso.

\section{O B S E R V C A O}

M.A., 55 anos de idade, sexo feminino, cor branca. Registro H.C. 1.011.038. Internada em 2-3-1973. História iniciada 5 anos antes, com desvio progressivo do globo ocular esquerdo para dentro e visão dupla. Dois anos e meio depois ocorreu ptose palpebral esquerda, que em 8 meses se tornou completa. Na mesma época a paciente notou que a hemiface esquerda estava menos sensivel que a direita, surgindo, 5 meses após, queda da acuidade auditiva ipsolateralmente. Um ano antes da internação a hemiface esquerda se tornou totalmente insensivel, havendo também piora da audição e início de atrofia da hemilíngua esquerda. Seis meses depois a paciente começou a sentir dificuldade para deglutir alimentos liqüidos, engasgando com freqüência, e 4 meses após notou que a boca se desviara para a direita, comecando a ter sensações vertiginosas. Exame clinico-neurológico - Pressão arterial $140 \times 90 \mathrm{~mm} \mathrm{Hg}$; pulso 88 batimentos por minuto; afebril; os exames clínico geral e especial nada revelaram de anormal, a não ser no que diz respeito ao comprometimento de nervos cranianos exclusivamente à esquerda: amaurose (fundos oculares sem papiledema e com pulso venoso presente); oftalmoplegia completa; anestesia térmica, tátil e dolorosa cutânea, assim como da bochecha, lábios e córnea da hemiface; desvio da mandibula para a esquerda ao abrir a boca; atrofias dos músculos masseter e temporal; paralisia facial periférica; hipoacusia e hiporreflexia vestibular;

Trabalho da Clínica Neurológica do Departamento de Neuropsiquiatria, Faculdade de Medicina da Universidade de São Paulo: * Preceptor; ** Assistente-Doutor; *** Assistente-Docente, Chefe do Setor de Neurorradiologia. 
diminuicão de reflexo nauseoso e presença do sinal da cortina; discretos déficit e atrofia dos músculos esternoclidomastóideo e trapézio; atrofia da hemilingua. Exames complementares - Craniogramas: calcificações de reduzidas dimensões ao nível do buraco óptico esquerdo; erosão do dorso selar, assim como da apófise clinóide posterior e da ponta da pirâmide esquerda. Eletromiografia dos músculos masseter $e$ temporal esquerdos: degeneração total das fibras. Encefalocintilografia com tecnécio: zona hipercaptante ao nível das fossas anterior e média esquerdas. Exame do $7 i$ qüido cefalorraqueano: normal. Carótido-angiografia esquerda: volumoso aneurisma da artéria carótida interna. Carótida-angiografia direita: aneurisma da região da comunicante anterior (figs. 1 e 2).
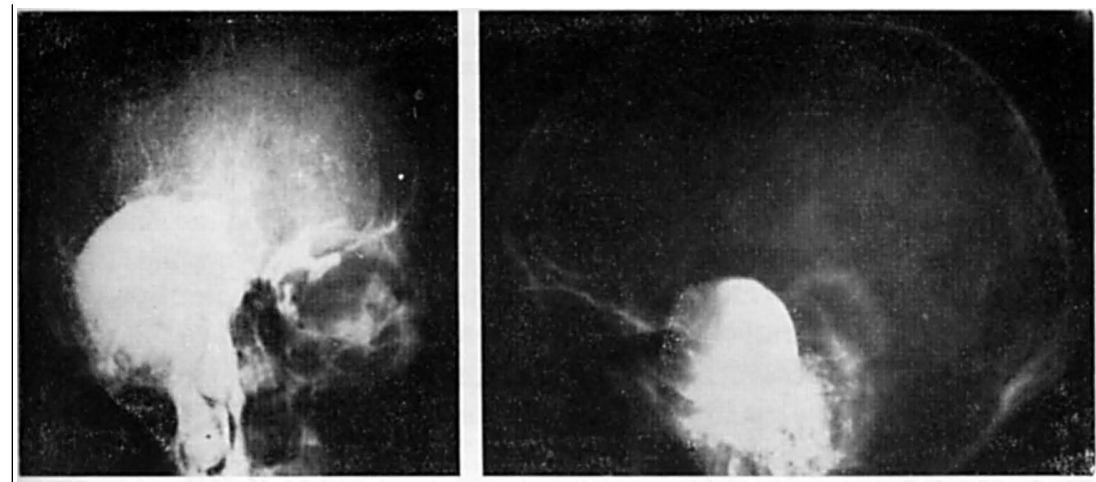

Fig. 1 - Caso M.A. - Angiografia pela carótida esquerda, mostrando volumoso aneurisma, que ocupa inteiramente a fossa média e parte da anterior.

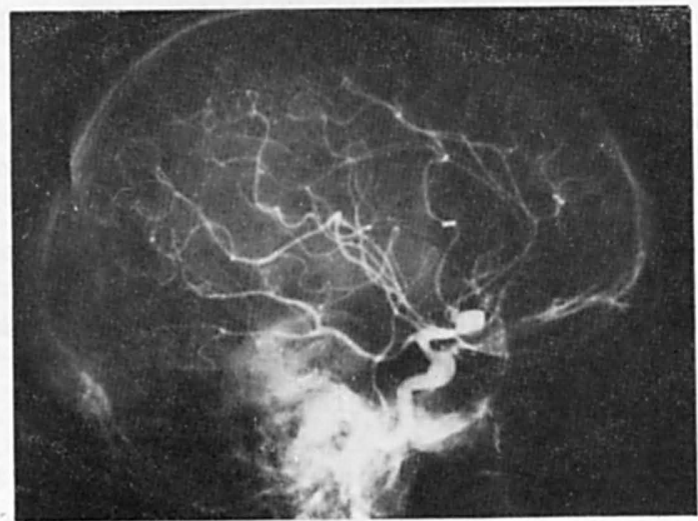

Fig. 2-Caso M.A. - Incidência lateral de angiografia pela carotídea direita, mostrando aneurisma na região da artéria comunicante anterior. 
Logo após o estudo angiográfico a paciente apresentou estado confusional e sinais de irritação meníngea, mostrando-se hemorrágico o líqüido cefalorraqueano. $O$ quadro clínico se agravou progressivamente, tendo a paciente falecido 4 dias depois.

Necropsia - Edema cerebral intenso, com engasgamento das amigdalas cerebelares. Hemorragia subaracnóidea recente. Aneurisma sacciforme da artéria carótida interna esquerda, que se iniciava imediatamente pós o seio cavernoso, com cerca de $5 \mathrm{~cm}$ de comprimento e $4,5 \mathrm{~cm}$ de largura. Erosão dos ossos da fossa média esquerda, principalmente da ponta da pirâmide e sela turca. O aneurisma apresentava intima relação com os nervos cranianos esquerdos e seus orifícios, comprimindo-os contra formações ósseas, máxime ao nível dos buracos redondo maior e oval. Foi encontrado aneurisma de médias dimensões $(1,5 \mathrm{~cm} \times 1,2 \mathrm{~cm})$ na artéria comunicante anterior. Este aneurisma não comprimia nervo craniano algum. Não foi possivel determinar qual dos aneurismas ocasionou o episódio hemorrágico.

\section{COMENTARIOS}

O comprometimento de nervos cranianos, configurando a sindrome de Garcin, é atribuído a várias etiologias, notadamente a neoplásica ${ }^{4}$. Em revisão da literatura, no entanto, não encontramos, entre as múltiplas causas desta síndrome, compressão provocada por aneurisma.

O nervo motor ocular comum é o mais comumente comprometido por aneurismas intracranianos $18,24,29$; por ordem de freqüência, seguem-se as vias ópticas pré-geniculadas 14, 15, 16, 18, 23, 24, 29; muito mais raramente observa-se o cometimento de outros nervos cranianos 1, 2, 7, 10, 17, 18, 21, 22, 29, 31.

$O$ fato de alguns aneurismas adquirirem grande volume sem se romperem ou provocarem hemorragia por diapedese está relacionado à estrutura de suas paredes e a condições hemodinâmicas ${ }^{24,29}$. Nos aneurismas gigantes têm sido descritos trombos laminares, que possivelmente reforçam a resistência de suas paredes $13,19,20,24,26,34$.

O estudo radiológico simples do crânio pode sugerir a presença destes aneurismas, evidenciando calcificaçōes múltiplas ou isoladas de suas paredes ${ }^{2}, 3,6,8,24,29$ e áreas de lise óssea, algumas vezes extensas, simulando processo neoplásico maligno. As áreas de osteolise se localizam de preferência, ao nivel das apófises clinóides anteriores e posteriores, assoalho da sela turca, fisura orbitária superior e canal da artéria carótida interna 3, 7, 24, 29.

A existência de aneurismas simulando neoplasias intracranianas torna insuficiente o estudo pré-operatório dos pacientes baseado apenas em pneumogramas e encefalocintilograma, exigindo a prática de angiografia 2, 8, 24, 26, 28, 29.

\section{R E S U M O}

E relatado caso de aneurisma gigante da artéria carótida interna esquerda, provocando síndrome de Garcin. O diagnóstico só pôde ser firmado mediante estudo angiográfico. A necropsia revelou aneurisma da artéria carótida interna com $5 \mathrm{~cm}$ por $4 \mathrm{~cm}$, comprimindo todos os nervos cranianos esquerdos, exceto o primeiro, e aneurisma da região da artéria comunicante anterior. 


\section{S U M M A R Y}

\section{Garcin's syndrome caused by a giant aneurysm of the internal carotid artery: a case report.}

A case of a large supra-clinoideal aneurysm of the left internal carotid artery involving from the second up to twelveth cranial nerves is reported. The patient, a 55-years-old white female, died 4 days after the angiographic study. The necropsy showed a giant aneurysm originated of the supraclinoideal left internal carotid artery, and an aneurysm of the anterior communicating artery.

\section{R E F E R N C I A S}

1. ALAJOUANINE, R. - La syntomatologie tumorale des volumineux aneurysmes des arteries vertebrales et basilares. Rev. neurol. (Paris) 80:321, 1948.

2. BULL, J. - Massive aneurysms at the base of the brain. Brain 92:535, 1969.

3. BULL, J. W. D. - Contributions of radiology to the study of intracrania] aneurysms. Brith med. J. ii:1701, 1962.

4. CANElas, H. M. \& JULIAO, O. F. - Paralisias múltiplas de nervos encefálicos produzidas por neoplasias da base do crânio. Arq. Neuro-Psiquiat. (São Paulo) 19:185, 1961.

5. CUATICO, W.; COOK, A. W.; TYSHCHENKO, V. \& ZHATIB, R. - Massive enlargement of intracranial aneurysms following carotid ligation. Arch Neurol. (Chicago) 17:609, 1967.

6. DANDY, W. E. - Intracranial arterial aneurysms in the carotid canal. Arch. Surg. (Chicago) 45:335, 1942.

7. DRAKE, C. G. - Management of aneurysms of posterior circulation. In R. J. Yoummans - Neurological Surgery. Saunders, Philadelphia, 1973, vol. 2, pp. 787-806.

8. DVORAK, M, \& KLAUS, E. - Schwierichkeiten in der Rötgendiagnostik und in der Behandlung von grossen intrakranialen sakulären Aneurysmen. Acta neurochir. (Wien) 15:182, 1966.

9. EPSTEIN, B. S. - The roentgenographic aspects of thrombosis of aneurysms of the anterior communicating and anterior cerebral arteries. Amer. J. Roentgenol. $70: 211,1953$.

10. HANDA, J.; KIKUCHI, H.; IWAYAMA, K.; TERAUDA, T. \& HANDA, H. Traumatic aneurysms of the internal carotid artery. Acta neurochir. (Wien) $17: 161,1953$.

11. HISKANNEN, O. \& NIKKI, P. - Large intracranial aneurysms. Acta neurol. Scand. 38:195, 1962 .

12. HYLAND, H. H. \& BARNETT, H. J. M. - The pathogenesis of cranial nerve palsies associated with intracranial aneurysms. Proc. roy. Soc. Med. 47:141, 1954.

13. JAIN, K. K. - Surgery of intracranial berry aneurysms: a review. Canada J. Surg. 8:172, 1965.

14. JEFFERSON, G. - On the sacular aneurysms of the internal carotid artery in the cavernous sinus. British J. Surg. 26:267, 1938.

15. JEFFERSON, G. - Chiasmal lesions produced by intracranial aneurysms. Arch. Neurol. Psychiat. (Chicago) 72:111, 1954.

16. JEFFERSON, G. - Further concerning compression of the optic pathways by intracranial aneurysms. In: R. K. Thompson - Clinical Neurosurgery. Willians \& Wilkins, Baltimore, 1955, Vol. 1, p. 55. 
17. KATF, N. Y. \& TOTLOW, W. F. T. - Two cases of vertebral aneurysms. Canad. Med. Ass. J. 92:471, 1965.

18. LE BEAU, J. \& BILLET, R. - Aneurysmes arteriels cerebrales. In Enciclopédie Médico-Chirurgicale, Neurologie, Vol. 5:7 49A-10, Paris, 1961.

19. LOCKSLEY, H. B. - Report on the cooperative study of intracranial aneurysms and subaracnoid haemorrhage. Section 5, part 1 - Natural history of subaracnoid haemorrhage, intracranial aneurysms and arterio-venous malformation. J. Neurosurg. 25:219, 1966.

20. LOCKSLEY, H. B. - Report on the cooperative study of intracranial aneurysms and subaraenoid haemorrhage. Section 5, part 2 - Natural history of subaracnoid haemorrhage, intracranial aneurysms and arterio-venous malformation. J. Neurosurg. 25:321, 1966.

21. LOUGHEED, W. M. \& BARNETT, H. J. M. - Lesions producing spontaneous haemorrhage. In R. J. Youmans - Neurological Surgery. Saunders, Philadelphia, 1973, Vol. 2, pp. 709-723.

22. MASSACHUSETTS General Hospital - Case records. New. Engl. J. Med. 271: 709, 1964 .

23. MILliSER, R. V.; GREENBERG, S. R. \& NEIMAN, B. H. - Congenital or berry aneurysms in the optic nerve. J. Clin. Path. 21:335, 1968.

24. MORLEY, T. P. - Giant intracranial aneurysms: diagnosis, course and management. In R. G. Ojemann - Clinical Neurosurgery. Willians \& Wilkins, Baltimore, 1969, vol. 16, pp. 73-94.

25. POOL, J. L. \& POTTS, D. G. - Aneurysms and Arteriovenous Anomalies of the Brain. Harper \& Row Publishers, New York, 1965.

26. SADIK, A. R.; BUDZILOVICH, G. N. \& SHULMAN, K. - Giant aneurysms of the middle cerebral artery. A case report. J. Neurosurg. 22:177, 1965.

27. SCHUNK, H. - Spontaneous thrombosis of intracranial aneurysms. Am. J. Roentgen 41:327, 1964.

28. STEHBENS, W. E. - Intracranial arterial aneurysms. Aust. Ann. Med. 3:214, 1954 .

29. Stehbens, W. E. - Pathology of Cerebral Vessels. Mosby, Saint Louis, 1972, pp. 351-470.

30. WALSH, F. B. \& KING, A. B. - Ocular signs of intracranial saccular aneurysms. Arch. Ophthal. (Chicago) 27:1, 1952.

31. WALtON, J. N. - Subaracnoid Haemorrhage. E. \& Livingstone, Edinburgo, 1956.

32. WHITE, J. C. \& ADAMS, R. D. - Combined supra and infraclinoid aneurysms of internal carotid artery. J. Neurosurg. 12:50, 1955.

33. WHITE, J. C. \& BALLANTINE, H. T. - Intraselar aneurysm simulating hypophyseal tumor. J. Neurosurg. 18:34, 1961.

34. YOUNG, G. \& FATTAL, G. A. - Arteriosclerotic aneurysms of the middle cerebral artery. Canada med. Ass. J. 89:720, 1963.

Clínica Neurológica - Faculdade de Medicina, Universidade de São Paulo Caixa Postal 3461 - 01000 São Paulo, SP - Brasil. 